\title{
Philosophiques
}

\section{Réponse à Don Ross}

\section{Denis Fisette et Pierre Poirier}

Volume 30, numéro 1, printemps 2003

Bernard Bolzano. Philosophie de la logique et théorie de la connaissance

URI : https://id.erudit.org/iderudit/007743ar

DOI : https://doi.org/10.7202/007743ar

Aller au sommaire du numéro

Éditeur(s)

Société de philosophie du Québec

ISSN

0316-2923 (imprimé)

1492-1391 (numérique)

Découvrir la revue

Citer cet article

Fisette, D. \& Poirier, P. (2003). Réponse à Don Ross. Philosophiques, 30(1),

256-262. https://doi.org/10.7202/007743ar

Ce document est protégé par la loi sur le droit d'auteur. L'utilisation des services d'Érudit (y compris la reproduction) est assujettie à sa politique d'utilisation que vous pouvez consulter en ligne.

https://apropos.erudit.org/fr/usagers/politique-dutilisation/ 


\title{
Réponse à Don Ross
}

\author{
DENIS FISETTE et PIERRE POIRIER \\ Université du Québec à Montréal
}

L'étude critique de Don Ross ne porte pas sur les paramètres généraux qui nous ont guidés dans cette étude, encore moins sur notre exposé des débats qui ont cours actuellement dans la philosophie de l'esprit et dans les sciences cognitives, mais elle porte principalement sur l'approche qui se profile dans les sept chapitres de l'ouvrage et que nous présentons schématiquement dans la conclusion. Son étude de cet aspect de l'ouvrage est particulièrement perspicace et nous croyons que Ross a très bien compris les enjeux, la portée mais aussi les limites de notre ouvrage. Puisqu'il manifeste une certaine sympathie à l'égard de notre évaluation critique de l'état des lieux dans la philosophie de l'esprit, la question qu'il pose n'est pas de savoir si un programme qui conjugue réalisme écologique et psychologie évolutionniste et qui met l'accent sur l'enchâssement corporel est viable, mais bien si ce programme entraîne un changement radical de régime ou une transformation en profondeur du cadre conceptuel de la philosophie de l'esprit. Car même en souscrivant à la critique du représentationnalisme comme le fait Ross, deux options peuvent être envisagées : le libéralisme, qui préconise un changement radical de régime, et le conservatisme à tendance œcuménique qui croit pouvoir s'accommoder aux modifications que nécessitent ces objections tout en restant sur ses positions. L'étude de Ross est en fait une mise en garde contre les dérapages ou "relâchements de l'esprit » auxquels pourrait conduire l'adhésion à l'option libérale. En réponse à Ross, nous ne chercherons pas à défendre l'une ou l'autre de ces options, mais nous voudrions évaluer les enjeux philosophiques qui sont associés à l'une et l'autre de ces options.

Commençons d'abord par désamorcer un préjugé tenace dont sont victimes plusieurs lecteurs de l'ouvrage, dont Don Ross. Il porte sur la signification des termes «phénoménologie » et "phénoménologues» et sur l'usage que nous en faisons dans l'ouvrage. À la fin de son étude, Ross indique que ses réserves à l'endroit de la forme de libéralisme que nous préconisons concernent au premier chef la phénoménologie et plus précisément cette phénoménologie que nous associons à l'idée d'un esprit incarné, mais que Ross, lui, semble identifier à des «métaphores vaguement hégéliennes ». C’est ce que confirme une autre remarque, lancée au tout début de son étude, alors qu'il discute de notre point de départ historique et qu'il interprète notre distinction d'une double voie vers la philosophie de l'esprit contemporaine de la manière suivante : «l'une phénoménologique-idéaliste, l'autre représentationnelle-réaliste ». D'où, semble-t-il, l'association possible entre la position que nous défendons dans l'ouvrage (et que nous qualifions de phénoménologique) et l'idéalisme «vaguement » hégélien! Nous croyons en effet que seul le préjugé qui identifie phénoménologie et idéalisme a pu nourrir de tels soupçons. Quelques remarques historiques suffiront peut-être à le désamorcer. 
1) Notre point de départ historique dans l'introduction de l'ouvrage est limité à cette version de la phénoménologie qu'on appelle la psychologie descriptive. Elle inclut aussi bien le jeune Husserl (jusque dans les Recherches logiques, 1901) que Brentano et ses étudiants Meinong, Twardowski, Stumpf et Marty, par exemple. Cette phénoménologie est non seulement étrangère à l'idéalisme classique, mais elle est, aux yeux de TOUS ses défenseurs, anti-idéaliste. C'est pourquoi nous avons pris soin de distinguer deux courants opposés du dix-neuvième siècle qui sont issus de Kant : le courant idéaliste, auquel appartient Hegel, et le courant psychologiste et réaliste auquel appartient Herbart, par exemple. Seul ce dernier présente un intérêt pour notre version de la phénoménologie.

2) Qu'est-ce qui justifie ce point de départ? Il s'agit d'abord et avant tout d'un choix stratégique et il est motivé par notre conviction que les questions fondamentales de la philosophie de l'esprit contemporaine sont très proches de celles qui étaient au cœur des préoccupations philosophiques des pères de la philosophie du vingtième siècle. La question du rapport entre la philosophie et la psychologie naissante à la fin du dix-neuvième siècle, c'est-à-dire de la psychologie comme discipline scientifique telle que nous la connaissons aujourd'hui, de même que les débats autour du psychologisme et ses conséquences sur la théorie de la connaissance durant la première moitié du vingtième siècle, nous a semblé la manière la plus simple et la plus directe d'expliquer la résurgence d'intérêt depuis Quine pour le domaine de la philosophie de l'esprit. À cet égard, rappelons ici les deux hypothèses historiques qui nous guident dans l'ouvrage :

Hypothèse 1 : La question philosophique qui rend possible, sur le plan historique, la connexion entre le contexte philosophique de la fin du dix-neuvième siècle et la philosophie de l'esprit contemporaine est le psychologisme.

Nous tenons pour acquis d'entrée de jeu que les arguments antipsychologistes de Frege et de Husserl contre les prétentions philosophiques de la psychologie scientifique ont eu pour conséquence, chez les empiristes logiques en particulier, de jeter un discrédit sur la valeur philosophique de la psychologie. D'où l'idée que philosophie et psychologie n'ont pas fait bon ménage durant toute la première moitié du vingtième siècle. Par psychologisme, que nous utilisons dans l'ouvrage dans un sens non péjoratif, nous entendons tout simplement la thèse suivant laquelle la psychologie occupe à elle seule tout le champ de la théorie de la connaissance.

La deuxième hypothèse, beaucoup plus complexe que la première, vise à rendre compte des conditions (historiques et théoriques) qui ont rendu possible le développement de la philosophie de l'esprit depuis le début des années soixante :

Hypothèse 2 : Que l'essor de la philosophie de l'esprit contemporaine a été rendu possible par deux facteurs importants : le développement des sciences cognitives et l'avènement du naturalisme philosophique. 
C'est la deuxième partie de cette hypothèse qui nous concerne plus particulièrement ici, soit l'avènement du naturalisme philosophique. Sur le plan historique, cette hypothèse nous semble porteuse puisqu'elle explique, croyons-nous, comment et à quelles conditions s'est faite la réhabilitation du statut philosophique de la psychologie contre les arguments antipsychologistes de Frege et de Husserl qui, rappelons-le, ont conservé toute leur force jusqu'à Quine. Ces conditions sont celles-là mêmes qui ont rendu possible l'implantation du programme d'une épistémologie naturalisée et au chapitre 3 de l'ouvrage, nous mentionnons trois de ces conditions : a) la critique de la dichotomie analytique/synthétique; b) la thèse du remplacement (replacement) ; 3) la critique du fondationalisme.

3) Notre point de départ dans la phénoménologie descriptive trouve aussi une nouvelle justification dans le rapport étroit que l'on peut établir entre le cadre conceptuel que l'on doit à cette tradition et l'appareil conceptuel dont se servent les philosophes de l'esprit contemporains. Nous nous contenterons ici de mentionner le concept d'intentionnalité qui, comme on le sait, est issu de cette tradition, et de renvoyer le lecteur aux chapitres 5 et 6 qui traitent respectivement de l'intentionnalité et de la rationalité.

4) En résumé, nous croyons qu'il existe un lien naturel entre cette phénoménologie et la philosophie de l'esprit contemporaine tant sur le plan épistémologique que sur le plan ontologique. En cela, notre point de départ n'a pas seulement une valeur stratégique dans l'ouvrage, mais il nous permet d'éviter les dérapages auxquels Ross fait allusion. Mais alors, rétorquera-t-on, puisque cette phénoménologie présente autant d'affinités avec la philosophie de l'esprit, du moins telle que vous l'entendez, pourquoi échapperait-elle aux critiques qui sont adressées au représentationnalisme dans la dernière partie de l'ouvrage? Autrement dit, qu'est-ce qui justifie l'opposition entre deux conceptions de l'esprit, plus précisément l'opposition du chapitre 7 entre deux attitudes face au problèmes de la conscience, entre le représentationnalisme et la phénoménologie? Car si l'on entend par représentationnnalisme cette conception de l'esprit qui repose uniquement sur le postulat d'états mentaux (ou phénomènes psychiques), alors cette phénoménologie qui nous sert de point de départ tombe elle aussi sous le coup de notre critique du théâtre cartésien. Il aurait donc fallu expliquer la relation entre cette version de la phénoménologie et celle plus récente que nous utilisons à la fin de l'ouvrage et qui s'apparente aussi bien à la psychologie de la forme et à la psychologie écologique qu'à ceux qui, dans la philosophie contemporaine, mettent l'accent sur l'aspect phénoménal de la conscience. Faute de pouvoir fournir une telle explication, et surtout parce que nous étions davantage préoccupés par le problème du fossé dans l'explication de la conscience (phénoménale), nous nous sommes penchés, dans le chapitre 7 , sur les nombreux arguments contre une forme ou une autre de repré- 
sentationnalisme et nous avons exploré, dans le chapitre suivant, une voie possible pour résoudre ce problème. Pour ce faire, nous nous sommes servis de la méthode naturelle de Flanagan parce que, comme lui, nous croyions que la solution au problème nécessitait le concours de la phénoménologie, des neurosciences et de la psychologie. Mais nous nous sommes passablement éloignés de cette version de la méthode naturelle en rapprochant la phénoménologie de la psychologie écologique, par exemple, et nous nous sommes demandés à quoi pourrait bien conduire un analogon de la méthode naturelle qui adopterait cette phénoménologie comme cadre descriptif. C'est en réponse à cette question que nous avons abouti aux programmes qui allient phénoménologie et sciences cognitives incarnées et situées (exprimées dans le vocabulaire propre à la théorie des systèmes dynamiques). Notre propos est forcément programmatique, mais nous avons indiqué on ne peut plus clairement qu'un tel programme sera réaliste ou ne sera pas. Critiquer le naturalisme brut et le réalisme métaphysique, ce n'est donc pas adhérer à une forme d'idéalisme contre laquelle Ross nous met en garde, mais c'est bien plutôt indiquer une troisième voie que d'autres appellent « réalisme direct » ou « réalisme naturel».

Venons-en maintenant à la question que nous avons énoncée au début de cette réponse, à savoir quel type de changement nous préconisons, ou du moins anticipons, concernant le cadre conceptuel de la philosophie de l'esprit : un changement radical ou simplement une transformation en profondeur. Ross voit bien que nous croyons que tout ne va pas pour le mieux en philosophie de l'esprit et qu'un changement s'impose, mais il manifeste, non sans raison, une certaine perplexité face au type de changement que nous préconisons ou anticipons. Bien que ce constat ne soit pas partagé par tous nos collègues, Ross et nous-mêmes reconnaissons que les différentes disciplines intéressées par l'esprit humain, qu'elles soient scientifiques (notamment les sciences biologiques, les neurosciences et sciences cognitives) ou philosophiques, nous ont suffisamment appris sur la nature de l'esprit pour voir dans la conception philosophique de l'esprit caractéristique du statu quo une option paralysante qui, nonobstant l'utilité qu'elle aurait pu avoir à une certaine époque, aveugle désormais les sciences cognitives : elle les lance à la recherche de solutions à des faux problèmes et leur fait ignorer les vrais questions ainsi que la manière adéquate de les résoudre. Mais cela dit, préconisons-nous un changement de cap radical ou simplement un coup de barre pour corriger une tendance un peut trop prononcée en philosophie de l'esprit? La perplexité de Ross s'explique, comme il le dit, par le fait que, pendant sept long chapitres, nous présentons la position traditionnelle de manière passablement bienveillante, ce qui suggère que nous espérons y trouver des éléments à conserver, mais que, dans notre conclusion, nous nous lançons dans une attaque en règle de la position traditionnelle et proposons des options qui pourraient la remplacer, ce qui n'est pas sans rappeler la fameuse thèse du 
remplacement de Quine dont nous traitons justement à cette fin dans notre ouvrage

Il y a d'abord deux raisons banales qui expliquent cette tension, ainsi que la perplexité qu'elles peuvent causer, et il convient de les mentionner ici, mais sans plus. D'abord la question est éminemment empirique, et nous croyons que ce n'est pas le rôle du philosophe que d'anticiper les développements de la science. Tout au plus notre tâche est de baliser les options qui s'offrent au chercheur et de souligner les présupposés théoriques ainsi que les conséquences de chacune. Ensuite, notre ouvrage se veut à la fois pédagogique et critique, et le premier objectif nous oblige à présenter la position traditionnelle de la manière la plus objective et bienveillante possible.

Toutefois, la tension dont fait état Ross résulte également des enjeux profonds du changement que nous anticipons et c'est de ces enjeux dont nous parlerons ici. Rappelons d'abord le mot de Searle, que souligne également Ross, à l'effet que la philosophie de l'esprit est maintenant devenue philosophie première et soulignons également que c'est la conception de l'esprit que nous qualifions ici de traditionnelle qui lui a conféré ce statut. Les raisons en sont multiples et, comme nous les avons longuement abordées dans notre ouvrage, nous n'en traiterons pas davantage. Il est plus important ici de remarquer que le changement qui s'impose selon Ross et nous-mêmes aura l'une ou l'autre de ces deux conséquences : ou bien celui-ci renverra la philosophie de l'esprit à son statut d'antan, soit une branche parmi d'autres de la philosophie, ou pire encore, c'est-à-dire sa disparition comme réflexion rationnelle ayant quoi que ce soit d'intéressant à dire sur l'esprit humain, ce discours ayant été pris en charge par les diverses sciences qui s'y intéressent déjà. Ou bien, comme le croient les Churchland (des radicaux, s'il en est), il imposera à la philosophie l'un de ces rares tournants l'amenant à revoir la discipline de fond en comble : qu'est-ce que la philosophie, quels problèmes sont proprement de nature philosophique et comment les résoudre. L'enjeu du changement, potentiellement, ne concerne donc pas uniquement l'étude philosophique et scientifique de l'esprit humain, mais toute la philosophie.

Mais revenons-en à la philosophie de l'esprit elle-même. Quels sont les enjeux des changements que nous anticipons en philosophie de l'esprit? Commençons par la position extrême, parce que, par son caractère radical, ses conséquences sont claires et faciles à énoncer. Elle consiste tout simplement à éliminer du champ des sciences cognitives la conception traditionnelle de l'esprit ainsi que toutes les recherches en sciences cognitives qui s'en sont inspiré. Cette position correspond à la thèse du remplacement de Quine ou à l'éliminativisme de Churchland et s'en distingue simplement par le choix du remplaçant : les sciences cognitives situées et incarnées (situated and embodied) associées à une certaine phénoménologie comme arrière-plan philosophique plutôt que le béhaviorisme de Quine et les neurosciences des Churchland. (Soit-dit en passant, nous croyons que la position radicale présentée dans notre ouvrage inclut les positions de Quine et de Churchland, 
ou du moins ce qu'on peut en rescaper, mais ne s'y limite pas.) Et comme le souligne Stich (1983) au début de son ouvrage qui est aussi éliminativiste à sa manière, l'élimination du schème par lequel on conçoit traditionnellement l'esprit aura au moins pour conséquence l'établissement d'une scission profonde entre l'image scientifique et l'image manifeste de l'homme, avec l'impossibilité de parvenir à la vision binoculaire que préconise Sellars (sinon une vision binoculaire où chaque œil voit un paysage entièrement différent et incommensurable). Et vu la position qu'occupe la philosophie de l'esprit dans le Zeitgeist actuel, cette scission aurait pour effet de consacrer la division profonde que certains voient entre la philosophie et les sciences. Au plus, et sans vouloir tomber dans l'optimisme exubérant dont font à l'occasion preuve les Churchland, l'éliminativisme pourrait avoir pour conséquence une redéfinition entière de la philosophie à l'image de la nouvelle science de la cognition. Nous en avons déjà parlé. Soulignons simplement ici que certains travaux vont déjà dans cette direction en épistémologie et en éthique (et il convient de souligner que ces travaux sont principalement avancés et développés par les Churchland eux-mêmes). Mais nous n'en sommes pas là. D'autant plus que l'option radicale n'est constituée pour l'instant que d'éléments choisis de la tradition phénoménologique et d'ébauches de programmes de recherche qui n'en sont encore qu'à l'état de métaphores. Pour qu'elles dépassent cet état, il faudra que les sciences cognitives contemporaines réussissent à rendre opérationnel ces métaphores et donner de la substance aux ébauches.

Étant donné ce que nous venons de dire au sujet de la perspective radicale, la position œcuménique pourrait sembler plus sage. Nous entrevoyons cependant deux problèmes possibles. D'abord, comme le dit le dicton, qui embrasse trop mal étreint. Il est possible qu'en voulant conserver certains éléments du schème traditionnel, on leste les nouvelles perspectives de recherche d'un fardeau qui les conduirait inéluctablement à leur échec. À cet effet, la position méthodologique que défend Dennett au sujet des qualia et qu'on pourrait qualifier d' "éliminativisme heuristique ", pourrait s'avérer plus profitable : n'imposons pas la conception traditionnelle aux nouvelles sciences de la cognition mais laissons-les plutôt se développer comme elles l'entendent. S'il s'avère que le produit final contient quelque chose de passablement semblable à la conception traditionnelle, alors nous saurons que celle-ci contenait un germe de vérité; dans le cas contraire, nous pourrons simplement constater le fait de son élimination. Ensuite, la position conservatrice est difficile à cerner. Par exemple, tous s'accordent sur le fait que la représentation est un élément essentiel de la conception traditionnelle et certains chercheurs radicaux ont vu leurs travaux comme la consécration d'une position anti-représentationnaliste (Brooks, Van Gelder, Dreyfus). Mais, comme même Brooks a dû le reconnaître éventuellement, ses recherches ne visent pas tant l'élimination de la représentation que celle d'une certaine conception de la représentation ainsi que de son rôle central dans la cognition. 
Mais qu'est-ce que constitue la position traditionnelle? Est-ce simplement l'usage d'un certain concept de représentation? Dans ce cas, la position de Brooks serait conservatrice. Ou encore, la position traditionnelle peut-elle être caractérisée par une conception particulière de la représentation, propositionnelle et symbolique par exemple, et par le rôle central qu'elle attribuerait à cette conception de la représentation au sein la cognition ? Dans ce cas, une position comme celle de Brooks serait pour nous radicale. Ainsi les termes « libéral» et «conservateur» ne caractérisent pas une dichotomie mais les pôles du continuum. Et nous avons clairement montré nos couleurs dans la conclusion de l'ouvrage : lorsque la poussière sera retombée, on pourra constater que le changement vécu dans les sciences cognitives et dans la philosophie de l'esprit sera beaucoup plus proche du pôle libéral que de son opposé. 